\title{
The Nottingham Fatigue After Stroke (NotFAST) Study: Results from follow-up six months after stroke
}

\section{Abstract}

2 Background: Post-stroke fatigue is common and disabling.

3 Objectives: The aim of NotFAST was to examine factors associated with fatigue in stroke

4 survivors without depression, six months after stroke.

5 Methods: Participants were recruited from four UK stroke units. Those with high levels of

6 depressive symptoms (score $\geq 7$ on Brief Assessment Schedule Depression Cards) or aphasia

7 were excluded. Follow-up assessment was conducted at six months after stroke. They were

8 assessed on the Fatigue Severity Scale, Rivermead Mobility Index, Nottingham Extended

9 Activities of Daily Living scale, Barthel Index, Beck Anxiety Index, Brief Assessment

Schedule Depression Cards, Impact of Event Scale-Revised, and Sleep Hygiene Index.

Results: Of the 371 participants recruited, 263 (71\%) were contacted at six months after stroke and $213(57 \%)$ returned questionnaires. Approximately half $(\mathrm{n}=109,51 \%)$ reported fatigue at six months. Of those reporting fatigue initially $(n=88), 61(69 \%)$ continued to report fatigue. 'De novo' (new) fatigue was reported by $48(38 \%)$ of those not fatigued initially. Lower Nottingham Extended Activities of Daily Living scores and higher Beck Anxiety Index scores were independently associated with fatigue at six months.

Conclusions: Half the stroke survivors reported fatigue at six months post-stroke. Reduced independence in activities of daily living and higher anxiety levels were associated with the level of fatigue. Persistent and delayed onset fatigue may affect independence and participation in rehabilitation, and these findings should be used to inform the development of appropriate interventions. 


\section{Keywords}

24 CVA; fatigue; follow-up; mood; rehabilitation; stroke; anxiety. 
Post-stroke fatigue (PSF) is common and adversely affects participation in rehabilitation,

daily occupational performance, return to work, and quality of life (1-3). In a survey of unmet needs after stroke, $43 \%$ of respondents reported that they had inadequate support to manage their fatigue (4). Yet, despite being an important clinical issue, there is a dearth of evidencebased recommendations for the prevention, treatment and management of PSF (5).

The course of fatigue for individual stroke survivors may vary. A recent review reported that approximately one third of participants who reported PSF early after stroke (within the first three months) experienced fatigue resolution by 12 months. However, some (12-58\%) of those without PSF in the early stages of recovery subsequently developed fatigue during the excess of 36 months post-stroke (7).

The association between depressive symptoms and fatigue has been established $(7,8)$, but evidence for other factors associated with PSF is often conflicting. Therefore, the overall aim of the Nottingham Fatigue After Stroke (NotFAST) study was to identify factors associated with fatigue, in a sample of stroke survivors without depression.

In our previous study, we investigated the factors affecting fatigue at four to six weeks following stroke onset $(n=268)$; these results have been reported in detail elsewhere (9). In summary, $115(43 \%)$ participants reported fatigue, of whom $71(62 \%)$ identified this as a post-stroke symptom. Multivariate analysis, using the Fatigue Severity Scale as the outcome variable, found that pre-stroke fatigue, having a spouse/partner, lower Rivermead Mobility Index score, higher Brief Assessment Schedule Depression Cards score, and higher Beck 
48 The aim of the study was to investigate factors associated with fatigue at six months post-

49 stroke.

\section{Materials and methods}

Ethical approval was obtained (NHS Health Research Authority Research Ethics Committee 13/EM/0187) and all procedures followed were in accordance with their guidelines.

NotFAST was a multi-centre, longitudinal cohort study; the methodology has been reported previously (9). Participants were recruited from four UK inpatient stroke services (Nottingham University Hospitals, University Hospitals of Leicester, University College London Hospitals and Salford Royal Hospital) over an 18-month period. Eligible participants had a clinical diagnosis of first stroke, were aged 18 years or over, and gave written consent. Participants were ineligible if they were unable to read or speak English sufficient to complete questionnaires or had a documented diagnosis of dementia.

Participants were screened for dysphasia using the Sheffield Screening Test for Acquired Language Disorders (10), and for depressive symptoms using the Brief Assessment Schedule Depression Cards (BASDEC) (11). Where there was significant dysphasia, i.e. those scoring below the age-recommended thresholds (10), or a BASDEC score consistent with a diagnosis of depression ( $\geq 7)$ (11), participants were excluded.

Remaining participants were assessed on the following measures, four to six weeks following stroke onset, and again by postal questionnaire at six months after stroke:

The Fatigue Severity Scale (FSS) of the Fatigue Assessment Inventory - nine item version (12) was used to assess the severity of fatigue. Scores range from 7 to 63 , with higher scores indicative of greater fatigue. A score $>36$ was used to indicate clinically significant fatigue, based on previous research. (13) 
Mobility was assessed using the Rivermead Mobility Index (14) (score 0-15), and independence in activities of daily living (ADLs) using the Barthel Index (15) (score 0-20) for personal care, and the Nottingham Extended Activities of Daily Living scale (16) (score 0-22) for instrumental activities of daily living. Sleep was assessed using the Sleep Hygiene Index (17) (score 0-52), with higher scores indicative of poorer sleep practices. Mood and emotional factors were assessed using the Brief Assessment Schedule Depression Cards (BASDEC) (11) (score 0 to 21) to detect depressive symptoms, Beck Anxiety Inventory (18) (score 0-63) to measure anxiety, and the Impact of Event Scale - Revised (19) (score 0-88) to detect post-traumatic stress. Higher scores are indicative of greater depression, greater anxiety and greater distress arising from traumatic events, respectively.

In order to maximise return of six-month postal questionnaires, the research team; checked with each participant's general practitioner that they were still alive and at the same address; provided stamped addressed return envelopes: undertook follow-up telephone calls to prompt participants (if questionnaires were not returned within three weeks); and offered telephone or face-to-face support to aid questionnaire completion. Where returned questionnaires were incomplete, participants were contacted by telephone to collect any missing information.

\section{Statistical analysis}

Data analyses were undertaken using IBM SPSS Statistics software version 22 . Where $\leq 10 \%$ of data was missing for a measure, and participants were not contactable, the missing values were assigned the mean score of items that they had completed on the measure. If $>10 \%$ of answers for a measure were missing, this item was omitted from the analyses.

Pearson's chi-square (using Yates' Correction for Continuity where applicable) and t-tests were used to compare the characteristics of participants who completed questionnaires with 
those who did not. An explanatory model was developed whereby those variables that were statistically significant in univariate analyses $(p \leq 0.05)$ were entered into a multivariable linear regression model. A step-wise modelling procedure was followed to obtain a final model including only statistically significant $(\mathrm{p} \leq 0.05)$ variables.

\section{Results}

Of the 371 participants recruited to the NotFAST study, 263 (71\%) were sent questionnaires at six months post-stroke (Figure 1). Two hundred and thirteen (57\%) questionnaires were returned; $50(13 \%)$ withdrew or did not respond. The mean number of days post-stroke at which questionnaires were completed was 198 (SD 29.69, range 162 to 430).

[Figure 1: Study recruitment and retention]

Demographic and clinical characteristics of participants and those who did not return sixmonth follow-up questionnaires are presented in Table 1.

[Table 1: Demographic and clinical characteristics of participants who completed, and those who did not complete, six-month follow-up questionnaires]

The characteristics of those who completed follow-up were comparable to those who did not, except for age. Participants who completed follow-up were significantly older (68.8 years, SD 12.36) than non-respondents (62.8 years, SD 17.2) $(p=0.03)$.

The results of the completed questionnaire measures at initial assessment and at six months are shown in Table 2. NEADL and RMI scores were significantly higher at six months $(p<0.001)$ than at four to six weeks post-stroke. Mood and sleep hygiene measure scores at six months did not differ significantly from those at four to six weeks after stroke. 
[Table 2: Distribution of questionnaire scores for participants who completed six-month

117 follow-up]

Mean FSS scores were significantly higher at six months post-stroke than at four to six weeks $(p=0.002)$. However, the proportion of participants reporting significant fatigue at six months was $51 \%(n=109)$, which was not significantly different $(p=0.07)$ from the proportion at four to six weeks ( $\mathrm{n}=115,43 \%)$. Of those who reported fatigue initially $(\mathrm{n}=88), 61(69 \%)$ continued to report fatigue. A further $48(38 \%)$ of those who were not fatigued previously $(\mathrm{n}=125)$ reported 'de novo' (new) fatigue at six months $(p=0.02)$.

\section{Factors associated with fatigue at six months after stroke}

Univariate analysis found higher FSS scores at six months to be associated with lower scores on the RMI, NEADL and BI $(p<0.001)$ and with higher scores on the BASDEC, BAI, IES-R and SHI $(p<0.001)$ (Tables 3 and 4). No other demographic or clinical characteristics were significantly associated with FSS scores at six months.

[Table 3: Relationship between fatigue and continuous variables at six-month follow-up]

[Table 4: Relationship between fatigue and categorical variables at six-month follow-up]

\section{Factors independently associated with fatigue at six months after stroke}

Multiple linear regression analysis was conducted using FSS score as the dependent variable, and variables found to be significantly associated with fatigue in the univariate analyses as independent variables.

In the final model (Table 5), 33\% of the variance in FSS scores at six months was accounted for by lower NEADL scale scores and higher BAI scores at six months. There was no difference in the overall model when repeated with a priori factors (age and gender). 
[Tables 5: Multiple linear regression model for analysis of relationship between FSS score and other variables at six-month follow-up]

\section{Discussion}

We found that fatigue was common at six months post-stroke (51\%), but less so than reported by Schepers et al. (20) (64\%) and by van de Port et al. (3) (68\%). Both of these studies were conducted in rehabilitation settings, and both assessed fatigue at six months post-stroke using the FSS. However, neither of these studies excluded participants with high levels of depressive symptoms, which may account for the greater proportions of fatigue reported. Furthermore, Schepers et al. (20) also reported a higher proportion of participants with fatigue at study commencement (52\%) compared with those in our cohort when assessed four to six weeks after stroke (43\%) (9).

The prevalence of fatigue at six months was also greater in our study than reported by Duncan et al. (21) (22\%) and by Radman et al. (22) (30\%). This is likely to be due to methodological differences. Duncan and colleagues used a case definition interview to define clinically significant fatigue, rather than a multi-item scale, and Radman et al. included people with less severe strokes (NIHSS score $\leq 3$ ) than in our sample.

Whilst we found the severity of fatigue to be greater at six months than at four to six weeks (9), the frequency of clinically significant fatigue was not significantly greater. A high proportion $(69 \%)$ of those fatigued in the early stages of recovery remained fatigued at six months. New cases of fatigue $(n=48)$ were reported, which is consistent with previous research (7). Our findings are broadly consistent with the time course of fatigue suggested by Wu et al. (6). Fatigue early in recovery may be a consequence of stroke-related biological factors, which have the potential to resolve, whilst longer-term fatigue may arise from chronic neurological deficit $(6,23)$. 
162 A lower level of independence in ADLs was a significant independent predictor of fatigue in the multivariate analysis, despite generally low levels of impairment overall. This finding differs from that reported by Van de Port et al. (3), who found no significant association between instrumental ADLs and fatigue at six months after stroke, after controlling for the influence of depression and impaired motor function. It may be that instrumental ADLs (e.g. shopping and social activities) have greater energy demands, and are more affected by fatigue, than basic ADLs (e.g. washing and dressing) (3). Although we excluded participants with symptoms consistent with a diagnosis of depression, it may be that even low levels of depressive symptoms, along with environmental and behavioural factors, contribute to reduced activity and participation in ADLs (24).

A strong statistical association was found between higher levels of anxiety symptoms and fatigue, which remained significant in the multivariate analysis. Other studies have reported a similar association between PSF and anxiety $(22,25,26)$. A recent meta-analysis of psychological associations with PSF identified a trend towards an association between fatigue and anxiety, but noted that, due to their co-morbid relationship, the presence of depressive symptoms may confound the reporting of anxiety (27). Psychosocial and behavioural factors may also play an important role in sustaining and mediating responses to fatigue $(6,21)$. Further studies to investigate factors which may underpin or sustain fatigue are required.

Our follow-up questionnaire return rate $(81 \%)$ was comparable to that of other studies using similar approaches $(1,28)$, although not all outcome measures were fully completed. However, where possible, the impact of this was mitigated by contacting the participants for clarification of missing data. There was an accidental omission of the BAI from eight questionnaire packs. Another limitation was that the study was not sufficiently powered to enable all possible factors related to fatigue to be investigated. 
Our choice of outcome measures for fatigue and anxiety may also be a limitation. The FSS is

187

188

189

190

191

192

193

194

195

196

197 commonly used in stroke research, however there is no validated 'cut-off' score to define clinically significant fatigue after stroke. Yet the approach we used to define significant fatigue is consistent with other studies. Whilst it is possible that the association between fatigue and anxiety symptoms reflects the overlap between stroke symptoms and descriptions of physiological anxiety symptoms used in the BAI, our findings regarding anxiety are nonetheless consistent with those reported by others.

\section{Conclusions}

At six months after stroke, fatigue was common in those who had experienced minor to moderate stroke. In some cases, this persisted from the early stages of recovery, whilst for others it was new 'de novo fatigue. Fatigue was associated with reduced independence in ADLs and higher levels of anxiety symptoms. The persistence of fatigue at six months, and the potential for delayed onset of fatigue, has important clinical implications for participation and the recovery of stroke survivors in the long-term. These findings indicate that levels of fatigue should be reviewed and interventions to address fatigue should be considered. Future research should conduct further validation studies on the FSS as a measure of clinically significant fatigue after stroke. In addition, research is needed to identify whether treatments to reduce depression and anxiety, and increase levels of independence in activities of daily living, have an effect on levels of fatigue.

\section{Acknowledgements}


208 We acknowledge the assistance of Emily Birks, Ella Clark, Christodouli Lagogianni, Holly

209 Milligan, Caroline Watchurst and Anne Worthington and also the support of the National

210 Institute of Health Research Clinical Research Network (NIHR CRN). We gratefully

211 acknowledge the input of our Steering Group, which comprised the authors, Dr Allison

212 Cooper, Mr Martin Kelly, Dr Peter Knapp, Professor Gillian Mead, Mr Ciaran Morgan, and

213 Dr Alex Pollock and the input of Dr Andrea Venn who provided statistical advice.

214

215 Declaration of interest statement

216 Disclosure of interest

217 The authors report no conflicts of interest.

218

219 Funding details

220 This research was supported by the Stroke Association under Grant TSA 2012/04.

221

222 Word count: 3,170 


\section{References}

1. Andersen G, Christensen D, Kirkevold M, Johnsen S. Post-stroke fatigue and return to work: a 2-year follow-up. Acta neurologica Scandinavica. 2012;125:248-53.

2. Morley W, Jackson K, Mead G. Post-stroke fatigue: an important yet neglected symptom. Age and Ageing 2005;34(3):313.

3. van de Port I, Kwakkel G, Schepers V, Heinemans C, Lindeman E. Is fatigue an independent factor associated with activities of daily living, instrumental activities of daily living and health related quality of life in chronic stroke? Cerebrovasc Dis. 2007;23:40-5.

4. McKevitt C, Fudge N, Redfern J, et al. Self-Reported Long-Term Needs After Stroke. Stroke. 2011;42:1398-403.

5. Wu S, Kutlubaev M, Chun $\mathrm{H}$, et al. Interventions for post-stroke fatigue. Cochrane Database of Systematic Reviews 2015(7).

6. Wu S, Mead G, Macleod M, Chalder T. Model of Understanding Fatigue After Stroke. Stroke. 2015;46(3):893-8.

7. Duncan F, Wu S, Mead G. Frequency and natural history of fatigue after stroke: a systematic review of longitudinal studies. J Psychosom Res. 2012;73:18-27.

8. Lerdal A, Bakken L, Kouwenhoven S, et al. Poststroke fatigue - a review. J Pain Symptom Manage. 2009;38:928-49.

9. Drummond A, Hawkins L, Sprigg N, et al. The Nottingham Fatigue After Stroke (NotFAST) Study: Factors associated with severity of fatigue in stroke patients without depression. Clinical Rehabilitation. First published $1^{\text {st }}$ March 2017. $\underline{10.1177 / 0269215517695857}$

10. Synder D, Body R, Parker M, Boddy M. Sheffield Screening Test for Acquired Language Disorders (Manual). Windsor: NFER Nelson; 1993.

11. Adshead F, Day Cody D, Pitt B. BASDEC : a novel screening instrument for depression in elderly medical inpatients. BMJ. 1992;305:397.

12. Schwartz J, Jandorf L, Krupp L. The measurement of fatigue: A new instrument. J Psychosom Res. 1993;37(7):753-62.

13. Lerdal A, Bakken LN, Rasmussen EF, et al. Physical impairment, depressive symptoms and pre-stroke fatigue are related to fatigue in the acute phase after stroke. ; (). Disabil Rehabil. 2011;33 (4):334-42. 
14. Collen F, Wade D, Robb G, Bradshaw C. The Rivermead Mobility Index: a further development of the Rivermead Motor Assessment. Int Disabil Stud. 1991;13:50-4.

15. Wade D, Collin C. The Barthel ADL index: a standard measure of physical disability. Int Disabil Stud. 1988;11:89-92.

16. Nouri F, Lincoln N. An extended activities of daily living scale for stroke patients. Clin Rehabil. 1987;1:301-5.

17. Mastin D, Bryson J, Corwyn R. Assessing sleep hygiene using the sleep hygiene index. J Behav Med. 2006;29(3):223-363.

18. Beck A, Steer R. Beck Anxiety Inventory (BAI) Manual. Oxford: Pearson; 1990.

19. Horowitz M, Wilner N, Alvarez W. Impact of Event Scale: a measure of subjective stress. Psychosom Med. 1979;41:209-18.

20. Schepers V, Visser-Meily A, Ketelaar M, Lindeman E. Poststroke Fatigue: Course and Its Relation to Personal and Stroke-Related Factors. Arch Phys Med Rehabil. 2006;87(2):184-8.

21. Duncan F, Lewis S, Greig C, et al. Exploratory Longitudinal Cohort Study of Associations of Fatigue After Stroke. Stroke. 2015;46:1052-8.

22. Radman N, Staub F, Aboulafia-Brakha T, Berney A, Bogousslavsky J, Annoni J. Poststroke fatigue following minor infarcts: a prospective study. Neurology. 2012;79(14):1422-7.

23. Kuppuswamy A, Rothwell J, Ward N. A model of poststroke fatigue based on sensorimotor deficits. Current opinion in neurology. 2015;28(6):582-6.

24. Barak S, Duncan PW. Issues in selecting outcome measures to assess functional recovery after stroke. NeuroRx: the journal of the American Society for Experimental NeuroTherapeutics 2006;3(4):505-24.

25. Glader E, Stegmayr B, Asplund K. Poststroke Fatigue: A 2-Year Follow-Up Study of Stroke Patients in Sweden. Stroke. 2002;33(5):1327-33.

26. Snaphaan L, van der Werf S, de Leeuw F. Time course and risk factors of post-stroke fatigue: a prospective cohort study. European journal of neurology. 2011;18(4):611-7.

27. Wu S, Barugh A, Mcleaod M, Mead G. Psychological associations of poststroke fatigue: a systematic review and meta-analysis. Stroke. 2014;45:893-8.

28. Parker C, Dewey M. Assessing research outcomes by postal questionnaire with telephone follow-up. Int J Epidemiol. 2000;29(6):1065-9. 